\title{
Performance of a Prototype RICH Detector Using Hybrid Photo-Diodes
}

\author{
E. Albrecht ${ }^{2)}$, G. Barber ${ }^{4)}$, J.H. Bibby ${ }^{5)}$, N.H. Brook ${ }^{3)}$, A. Duane ${ }^{4)}$, M. French ${ }^{6)}$, V. Gibson ${ }^{1)}$, \\ R. Giles ${ }^{5)}$, A.W. Halley ${ }^{2) 3)}$, N. Harnew ${ }^{5)}$, M. John ${ }^{4)}$, D. G. Miller ${ }^{4)}$, V.O'Shea ${ }^{3)}$, P. Teixeira-Dias ${ }^{3)}$, \\ N. Smale ${ }^{5)}$, D. Websdale ${ }^{4)}$, G. Wilkinson ${ }^{25)}$, S.A. Wotton ${ }^{1)}$.
}

\begin{abstract}
A prototype Ring-Imaging Cherenkov detector has been operated in a charged particle test beam. Cherenkov photons are imaged onto a plane of hybrid photo-diode detectors. The geometrical arrangement of the prototype and data-taking conditions are described. An analysis of the detector performance, using silica aerogel, air and $\mathrm{C}_{4} \mathrm{~F}_{10}$ gas radiators, is presented. The photon yields and observed Cherenkov angle resolutions are found to be in good agreement with Monte Carlo simulation and satisfy the requirements of the $\mathrm{RICH} 1$ detector in the $\mathrm{LHCb}$ experiment.
\end{abstract}

(to be submitted to NIM A)

1) University of Cambridge, Cavendish Laboratory, Madingley Road, Cambridge CB3 OHE, UK.

2) CERN, PPE Division, 1211 Geneva 23, Switzerland.

3) University of Glasgow, Department of Physics, Glasgow G12 8QQ, UK.

4) Imperial College of Science Technology \& Medicine, Blackett Laboratory, Prince Consort Road, London SW7 $2 A Z$, UK.

5) University of Oxford, Department of Nuclear Physics, Keble Road, Oxford OX1 3RH, UK.

6) Rutherford Appleton Laboratory, Chilton, Didcot, Oxon OX11 OQX, UK. 


\section{Introduction}

This paper reports results from a prototype Ring Imaging Cherenkov ( $\mathrm{RICH}$ ) counter which uses an array of hybrid photo-diode detectors (HPDs) to detect the Cherenkov photons. This is a prototype of the upstream RICH detector of the $\mathrm{LHCb}$ experiment [1]. The LHCb experiment is a singlearm spectrometer, recently approved to run at the CERN Large Hadron Collider. The data were collected during the spring and summer of 1997 at the CERN PS facility.

The LHCb experiment is designed to make precision measurements of $\mathrm{CP}$ violation in $B$ decays. The experiment utilises two $\mathrm{RICH}$ detectors. The upstream detector ( $\mathrm{RICH}-1)$ covers polar angles between approximately 25 and $330 \mathrm{mrad}$, and the downstream detector $(\mathrm{RICH}-2)$ covers polar angles between approximately 10 and $120 \mathrm{mrad}$. The RICH detectors are crucial in providing charged $\pi / \mathrm{K}$ separation for particles with momenta between 1 and $>100 \mathrm{GeV} / \mathrm{c}$. The detectors reduce backgrounds in selected final states, eg. $\mathrm{B}_{\mathrm{d}}^{0} \rightarrow \pi^{+} \pi^{-}, \mathrm{B}_{\mathrm{s}}^{0} \rightarrow \mathrm{D}_{\mathrm{s}}^{ \pm} \mathrm{K}^{\mp}$, hence minimising systematic errors on $\mathrm{CP}$ violation measurements. They are also used to provide an efficient flavour tag of $B$ mesons using kaons.

The RICH-1 detector $[1,2,3]$ combines gas and aerogel radiators to provide $\pi / \mathrm{K}$ separation over the momentum range $1-65 \mathrm{GeV} / \mathrm{c}$. The aerogel is placed against the entrance window, with a second $\mathrm{C}_{4} \mathrm{~F}_{10}$ gas radiator behind it. With a refractive index of $n=1.03$, a $5 \mathrm{~cm}$ thick slab of aerogel provides low momentum $\pi / \mathrm{K}$ separation up to $\sim 10 \mathrm{GeV} / \mathrm{c}$. It gives an expected mean number of $\sim 15$ detected photoelectrons for a fully saturated $(\beta=1)$ track. The $\mathrm{C}_{4} \mathrm{~F}_{10}$ gas radiator (with $n=1.0014$ ) is approximately $95 \mathrm{~cm}$ long, covers the momentum range up to $65 \mathrm{GeV} / \mathrm{c}$, and gives an expected mean number of detected photoelectrons of 55. A spherical mirror of focal length $1 \mathrm{~m}$ is common to both the gas and aerogel radiators. The mirror is tilted by $\sim 250 \mathrm{mrad}$ to reflect Cherenkov photons out of the spectrometer acceptance and onto the photodetector plane. The $\mathrm{RICH}-2$ radiator comprises approximately $180 \mathrm{~cm}$ of $\mathrm{CF}_{4}$ gas.

The LHCb RICH counters use arrays of photon detectors to observe the Cherenkov light. HPDs are the chosen baseline option for the LHCb photon detectors as they provide large-area coverage, whilst the silicon segmentation gives the necessary detector granularity [4]. HPDs have low noise, a high quantum efficiency and good single-photon sensitivity. However, when operated at $20 \mathrm{kV}$ with a gain of $\sim 5000$, the signal charge resulting from one photoelectron is relatively small. The devices used in the tests reported here were manufactured commercially by $D E P^{1)}$. They have an hexagonal array of 61 pixels. The pixels have an area of approximately

\footnotetext{
1) Delft Electronics Products BV, 9300AB Roden, NL.
}

$2 \times 2 \mathrm{~mm}^{2}$ which is the same as that proposed for LHCb, but with a much smaller active photocathode area of $\sim 190 \mathrm{~mm}^{2}$.

The aim of the prototype RICH tests described here is to measure, in a realistic beam environment, the following :

- The signal-to-noise ratio of the HPDs and whether they are able to detect the relatively small numbers of photons with an acceptable efficiency.

- Photon yields from aerogel, air and $\mathrm{C}_{4} \mathrm{~F}_{10}$ radiators and to compare that observed with expectations from theory and simulation. The aim is to ensure enough redundancy to allow robust pattern recognition in the full $\mathrm{LHCb}$ $\mathrm{RICH}-1$ detector.

- To reconstruct the Cherenkov angle, and understand the contributions to its measurement resolution. The aim is to determine the overall precision of the RICH so as to verify the $\pi / \mathrm{K}$ separation performance of the $\mathrm{LHCb}$ detector as a whole.

This paper presents detailed analyses of data from the RICH-1 prototype and comparisons with simulation. It is structured as follows. Section 2 briefly describes the features of the $\mathrm{RICH}-1$ prototype, its electronic readout and accompanying silicon telescope. Section 3 describes the data selection criteria and photon detector performance whilst Section 4 describes the technique of geometrical alignment of the individual elements. Details of the detector simulation are given in Section 5. An estimation of the photon yields from aerogel and gas radiators are described in Section 6. Section 7 presents the reconstruction of the mean Cherenkov angle for the aerogel and $\mathrm{C}_{4} \mathrm{~F}_{10}$ radiators, and contributions to the angular resolution of these measurements. Finally, a summary is given in Section 8, including plans for future work.

\section{$2 \quad$ Experimental arrangement}

This section briefly describes the experimental layout of the RICH-1 prototype detector. A full description of the apparatus, and the detector configurations used, can be found in [3].

The tests make use of two distinct geometrical configurations of detector prototype, referred to throughout as Configurations 1 and 2. Configuration 1 is a $\frac{1}{4}$ scale model of RICH-1. It is used with a total of 5, and later 7, HPDs to study the photon yield and Cherenkov angle resolution from aerogel and the photon yield from gas radiators. Configuration 2 is a full-scale prototype of the optical layout of $\mathrm{RICH}-1$ and is used to measure the Cherenkov angle resolution with the $\mathrm{C}_{4} \mathrm{~F}_{10}$ gas radiator. The geometry and characteristics of the two configurations are described below :

- Configuration 1 consists of a vessel of dimensions $800 \times 600 \times 600 \mathrm{~mm}$. The vessel is 
aligned such that the beam passes through a block of aerogel, followed by $400 \mathrm{~mm}$ of air or $\mathrm{C}_{4} \mathrm{~F}_{10}$ gas radiator. This is shown schematically in Figure 1. Cherenkov photons pro-

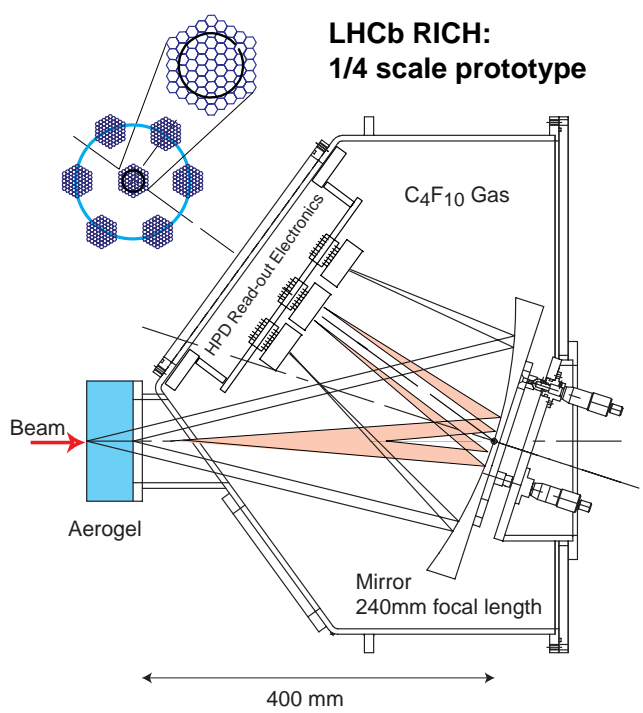

Figure 1: Schematic layout of Configuration 1, the $\frac{1}{4}$-scale prototype RICH-1 vessel.

duced are reflected and focused by the mirror onto an array of HPDs. These are mounted on movable elements, placed $246 \mathrm{~mm}$ from the mirror centre, at an $18^{\circ}$ tilt relative to the beam.

- Configuration 2 uses two extension tubes to increase the active gaseous radiator length of Configuration 1 to $1 \mathrm{~m}$. The mirror focal length is also increased to $1117 \mathrm{~mm}$. The geometry is shown schematically in Figure 2.

A three-dimensional coordinate system is defined for the purpose of reconstruction and alignment. The origin of the orthogonal axes is defined to lie at the point where the beam intersects the mirror surface. The $z$-axis then lies along the beam direction, pointing upstream. The $x$-axis is then defined to lie in the horizontal plane in the direction of the detector plane, with the $y$-axis pointing vertically upwards. The two detector configurations share common components which are :

- The radiators : Two types of silica aerogel samples are used, both with areas of $110 \times$ $110 \mathrm{~mm}^{2}$ perpendicular to the input beam direction. They are referred to here and in [3] as samples 1 and 2, with thicknesses of 18 and $11 \mathrm{~mm}$ respectively ${ }^{2}$. Their optical transmissions were measured in the laboratory and are shown in Figure $3(\mathrm{~d})$. The aerogel samples

\footnotetext{
2) Respectively manufactured by the KEK Laboratory, Japan, supplied by J.Seguinot, Collège de France, and by Matsushita Company, Japan, supplied by E. Nappi,
}

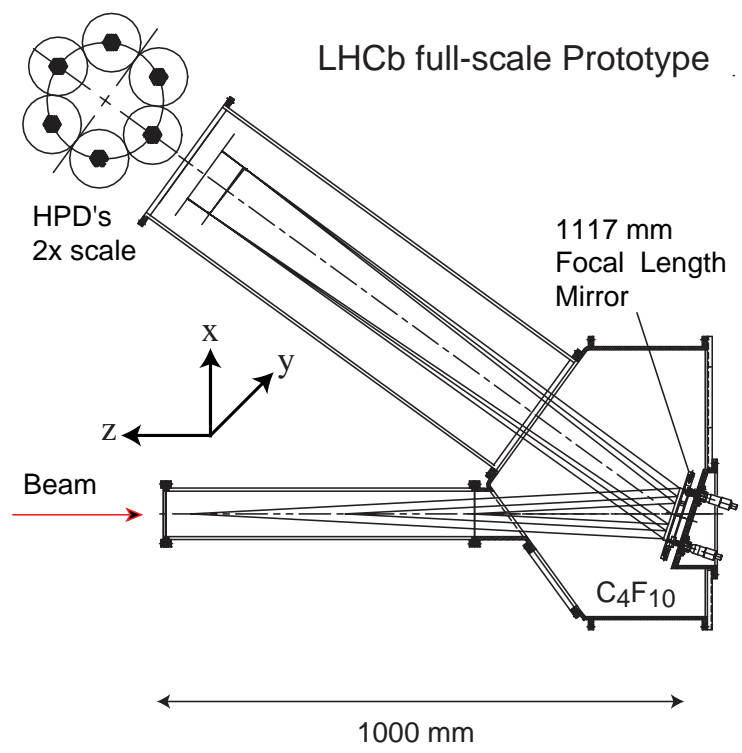

Figure 2: Schematic layout of Configuration 2, the full-scale prototype $\mathrm{RICH}-1$ detector.

have nominal refractive indices of $n \sim 1.03$, and are positioned $425 \mathrm{~mm}$ upstream of the mirror centre. Fits to the transmission curves shown in Figure 3(d), both return a clarity coefficient of $0.008 \mu \mathrm{m}^{4} / \mathrm{cm}$. The dimensions of the air and $\mathrm{C}_{4} \mathrm{~F}_{10}$ gas radiators in each configuration are given in Table 1 .

- The detector plane : The photodetector plane holds up to seven 61-pixel hybrid photo-diodes ${ }^{3)}$, contracted from the DEP company. These are run at a common acceleration voltage of between 10 and $12 \mathrm{kV}$ during normal operation [3]. The detectors are read out individually using VIKING VA2[5] amplifiers with a shaping time of $\sim 1.2 \mu \mathrm{s}$. The detectors and their electronics are mounted onto a plate where, after initial alignment, their $(x, y)$ positions and rotation angles are fixed. The orientation and numbering of the HPDs on the detector plane is indicated in Figure 4. Filters are placed over some of the HPD input windows to restrict the detected wavelength. In these tests, borosilicate glass and mylar filters are used.

- The mirrors: These are mounted on the backplate of the vessel and held on three micrometer screws. They are adjusted to align the Cherenkov ring image onto the centre of the detector plane. The focal lengths of the mirrors used in each detector configuration are given in Table 1.

University of Bari, Italy.

3) A product issuing from a collaborative development project with INFN (Italy) and Southampton University (UK), with funding from the European Union. 

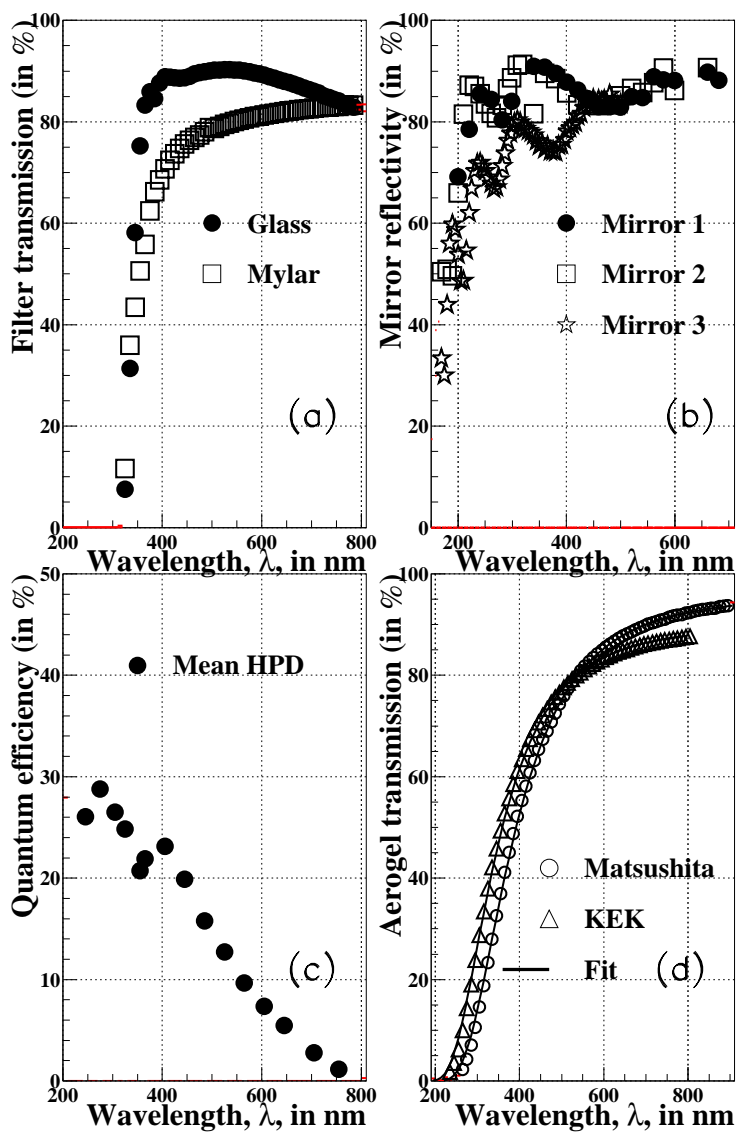

Figure 3: (a) Transmission characteristics for glass and mylar, (b) mirror reflectivity, (c) HPD detector quantum efficiency and (d) measured and fitted aerogel transmission curves as funct ion of wavelength.

- The gas circulation system : The $\mathrm{RICH}$ vessel is connected to a gas recycling and purification system [3] that cycles $\mathrm{C}_{4} \mathrm{~F}_{10}$ gas approximately once every hour. For these tests, a slight overpressure is maintained.

\begin{tabular}{|l|c|}
\hline Quantity & Value \\
\hline Mirror-to-beam Angle & $18( \pm 2)^{\circ}$ \\
\hline Mirror Focal Lengths : & \\
Configuration 1 & $236( \pm 2) \mathrm{mm}$ \\
Configuration 2 & $1117( \pm 10) \mathrm{mm}$ \\
\hline Mirror-to-detector distance : & \\
Configuration 1 & 245 or $250 \mathrm{~mm}$ \\
Configuration 2 & $1143( \pm 4) \mathrm{mm}$ \\
\hline
\end{tabular}

Table 1: Measured global geometry parameters for the three RICH configurations.

The prototype $\mathrm{RICH}$ vessel was sited in the CERN T9 beam, which is a secondary beam derived from extracted PS protons, and provides charged particles of either polarity. Particle momenta from

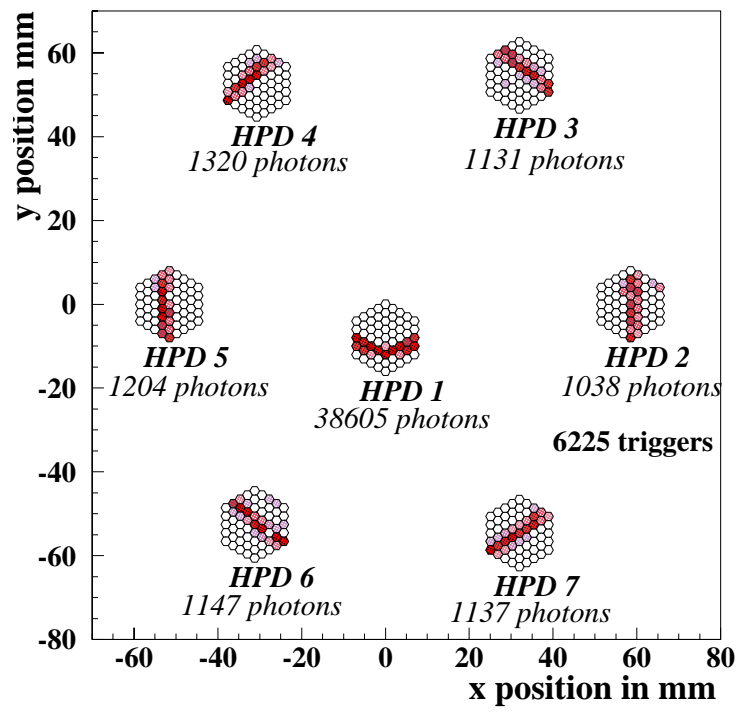

Figure 4: An event display shown on the front face of the detector plane of HPDs indicating the 7 detector positions. The ring is due to photons emitted in the $\mathrm{C}_{4} \mathrm{~F}_{10}$.

$2 \rightarrow 15.5 \mathrm{GeV} / \mathrm{c}$ are available. The particle type $(e$, $\pi, \mathrm{K}, \mathrm{p})$ can be separated using a $\mathrm{CO}_{2}$ threshold Cherenkov counter, installed $30 \mathrm{~m}$ upstream of the prototype.

Incident beam particles are selected using two upstream and two downstream scintillation counters. The last two are placed $4 \mathrm{~m}$ apart and the smallest scintillator $\left(4 \times 4 \mathrm{~mm}^{2}\right)$ defines the accepted beam size and an expected beam divergence of not more than $3 \mathrm{mrad}$. Scintillators 1 and 4 are $50 \times 50 \mathrm{~mm}$ and cover the whole beam. The readout is triggered by the four scintillation counters in coincidence. During a typical extraction pulse of $200 \mathrm{~ms}$ duration, $5 \times 10^{3}$ beam particles enter the detector volume, resulting in $\sim 30$ triggers.

In Configuration 2, the beam trajectory is measured using a silicon telescope. This consists of three planes of silicon-pad detectors. Each plane has $22 \times 22$ pads, with pad dimensions $1.3 \times 1.3 \mathrm{~mm}^{2}$. The planes are placed upstream of the prototype and separated by approximately $1 \mathrm{~m}$. The purpose of the telescope is to allow event-by-event track direction measurement from which the Cherenkov angle can be more precisely measured, giving a beam trajectory uncertainty of approximately 0.38 mrad. This assumes perfect geometrical alignment, and is discussed later in Section 4. Analogue signals from this detector are read out using the same digitiser as the HPDs, as described in [3].

\section{Data selection criteria}

On receipt of a trigger signal, the charge accumulated on each pixel of each HPD is read out, 


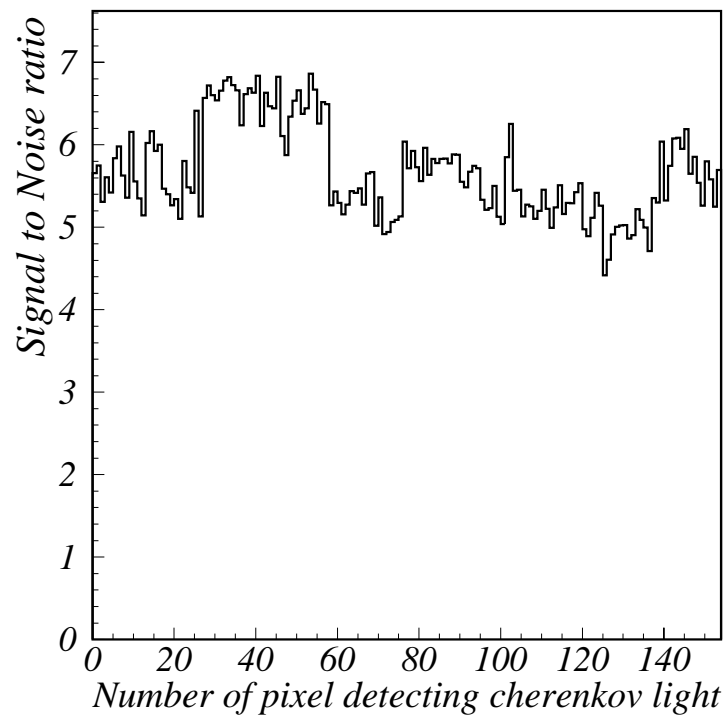

Figure 5: Signal-to-noise ratios for a subset of the HPD detector channels.

digitised by an ADC and written to disk. Events are then selected for data analysis by simply requiring that there is at least one "hit pixel". The threshold for a hit pixel is defined by requiring that the recorded ADC value for that channel lies more than a certain number of pedestal sigma ${ }^{4)}$ away from the pedestal mean. These thresholds are determined for each channel separately, and are calculated using dedicated pedestal runs which are interleaved between data-taking with beam.

The choice of threshold (or sigma cut) is primarily determined by the signal-to-noise response of the HPDs. The signal-to-noise of each pixel is determined by measuring the photon peak separation, from spectra obtained from dedicated runs with a pulsed LED, and comparing it with the width of the measured pedestal distribution. The results for approximately $\frac{1}{3}$ of the total channels are shown in Figure 5. The projection of this distribution is shown in Figure 6 and yields a mean of 5.7 and a spread of 0.6 for 181 pixels on 5 HPDs. The stability of the noise pedestals, observed over several weeks at a time, indicates that, for the majority of channels, any change in pedestal width with time is less than $7 \%$ of a typical photoelectron signal. In addition, $3 \%$ of the channels were dead due to the readout electronics and $0.7 \%$ noisy due to high leakage currents in the silicon.

The value used for the sigma cut on hit pixels is analysis dependent. It represents a compromise between the efficiency of photon selection and the purity of the sample obtained. Typically a 3 sigma

\footnotetext{
4) The sigma is defined as being the standard deviation from a Gaussian fit to the pedestal distribution.
}

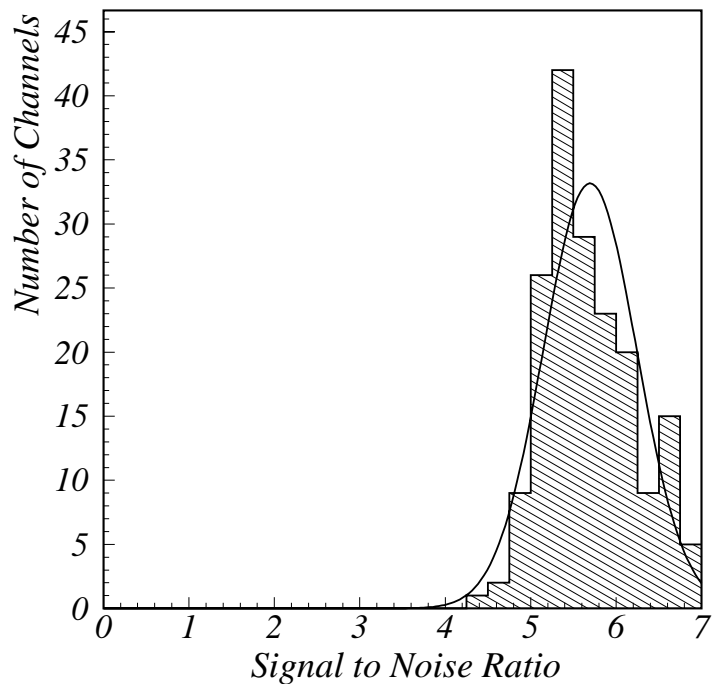

Figure 6: Distribution of channel-by-channel signalto-noise ratios for a subset of the HPD detector channels.

cut results in an efficiency of $\sim 85 \%$ for selecting signal photons, with a small background due to electronic noise. Cuts at 4 or 5 sigma are significantly less efficient but are effectively free of background. Variation of this selection is used to estimate systematic uncertainties.

The threshold Cherenkov detector is also used to estimate systematic uncertainties by selecting varying beam compositions. This is achieved by setting a threshold which determines the accepted kaon and antiproton contamination. The silicon telescope planes have a signal-to-noise ratio better than $20: 1$, and so a simple cut is made to select these with negligible contamination from noise.

\section{Alignment of the silicon telescope}

The beam telescope is used to provide an event-by-event track direction for the Cherenkov angle reconstruction. To achieve this, the alignment uncertainty of the telescope must be significantly less than 0.4 mrad with respect to the $\mathrm{RICH}$ vessel.

Internal alignment of the telescope is achieved by selecting events with precisely one hit in each plane and studying fit residuals with respect to a straight line. External alignment is performed using events with $\geq 2$ detected photons in the $\mathrm{RICH}$. For measured track slopes $\left(m_{x}, m_{y}\right)$ different from their true values :

$$
m_{x}^{\text {true }}=m_{x}+\delta_{m_{x}}, \text { and } m_{y}^{\text {true }}=m_{y}+\delta_{m_{y}} .
$$

then a shift of the measured Cherenkov angles is induced. By minimising the spread in the measured Cherenkov angles, as a function of $\left(\delta_{m_{x}}, \delta_{m_{y}}\right)$, their optimum values are determined as shown in Figure 7 . The values of $\delta_{m_{x}}$ and $\delta_{m_{y}}$ which minimize 


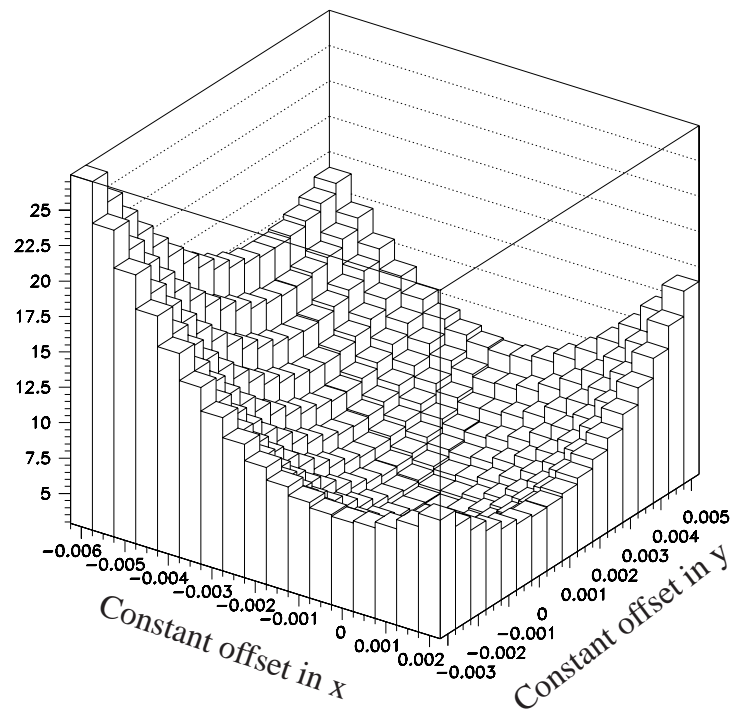

Figure 7: Variance of the measured Cherenkov angles as a function of the constant offsets, $\delta m_{x}$ and $\delta m_{y}$, applied to the gradient of the silicon telescope beam vector.

the average variance $\left\langle\sigma^{2}\left(\theta_{1}, \theta_{2}, \ldots, \theta_{\mathrm{n}_{\gamma}}\right)\right\rangle$ are of the order of $\pm 1 \mathrm{mrad}$, and are used to correct the event-by-event beam direction determined from telescope data.

\section{Simulation of the $\mathrm{RICH}-1$ prototype}

The RICH-1 prototype configurations are simulated to allow detailed comparisons of expected performance with data. The simulation is also used in the design of the prototypes themselves, to calculate the optimal position of the detector plane from the mirror. The program simulates photon emission in the radiators and assigns each a wavelength, Cherenkov angle and point of emission. Photons are then ray-traced to the HPD detectors and assigned to a hit. The program incorporates the following physical effects :

- Beam trajectory and composition : For each event, an input beam direction and position is defined according to the acceptance of the trigger scintillators. The input particle is defined to be either a $\pi^{-}, e^{-}$or $K^{-}$in ratios of the real beam composition. The PS beam delivers particles with a given, fixed momentum to within $\pm 1 \%$. The spread in momentum is also included in the simulation.

- Photon Generation and Chromatic Aberration : Photons emitted in the radiator are assigned an energy distribution ranging from $1.5 \mathrm{eV}$ to $7.3 \mathrm{eV}$ according to the relation :

$$
\frac{d N_{\gamma}}{d E}=370 \sin ^{2} \theta_{\mathrm{C}} L
$$

where $\theta_{\mathrm{c}}$ is the Cherenkov angle, $L$ is the radiator length in $\mathrm{cm}$ [4]. The dispersion of the refractive index is parameterised in the simulation by the Lorentz-Lorenz equation [6] :

$$
n(E)^{2}=\frac{1+2 c f(E)}{1-c f(E)}
$$

where :

$$
\begin{gathered}
c=\frac{4 \pi a \rho N_{A}}{2 M}=0.3738 \mathrm{~cm}^{3} \frac{\rho}{M} \\
f(E)=\frac{F_{1}}{G_{1}^{2}-E^{2}}+\frac{F_{2}}{G_{2}^{2}-E^{2}}
\end{gathered}
$$

and $\rho$ is the density of the medium, $M$ is molecular weight, $E$ is the photon energy and $F$ and $G$ are Sellmeir coefficients. The latter are derived quantities, examples of which are tabulated in reference [7]. In the simulation, air is approximated by nitrogen, aerogel by quartz [4] (scaled by their relative densities) and $\mathrm{C}_{4} \mathrm{~F}_{10}$ by $\mathrm{C}_{2} \mathrm{~F}_{6}[8]$ scaled to fit measured data [9]. Figure 8 shows the simulated behaviour of the refractive indices for aerogel and $\mathrm{C}_{4} \mathrm{~F}_{10}$ with respect to the wavelength of the emitted photon. Finally, the Cherenkov angle is generated with a uniform azimuthal angular distribution.

- Emission Point \& Beam Trajectory Uncertainty : In the LHCb RICH-1 counter, the mirror is split and tilted to direct the ring images out of the detector acceptance [1]. This introduces a photon emission point error. When reconstructing the Cherenkov angle, it is necessary to assume an emission point for the photons, which is taken to be the midpoint of the radiator.

The beam trajectory uncertainty is assumed to be either determined by the trigger scintillator acceptance in the absence of the silicon telescope, or that determined by the silicon telescope pixel size and the nominal $(0,0,-1)$ simulated beam direction is smeared accordingly.

- Ray tracing : Each photon is individually traced through the prototype geometry. The photon trajectory is modified by refraction at the gas/aerogel boundary, reflection from the mirror surface, absorption by the walls of the chamber or Rayleigh scattering in the aerogel. The chromatic dependence of the mirror reflection and transmission properties of the aerogel are used as measured, and are shown in Figures 3(b) and 3(d).

- Simulation of the HPD : The quantum efficiency of the HPD photocathode, as a function of photon wavelength, is supplied by the manufacturer and shown in Figure 3(c). From these data the simulation determines whether 


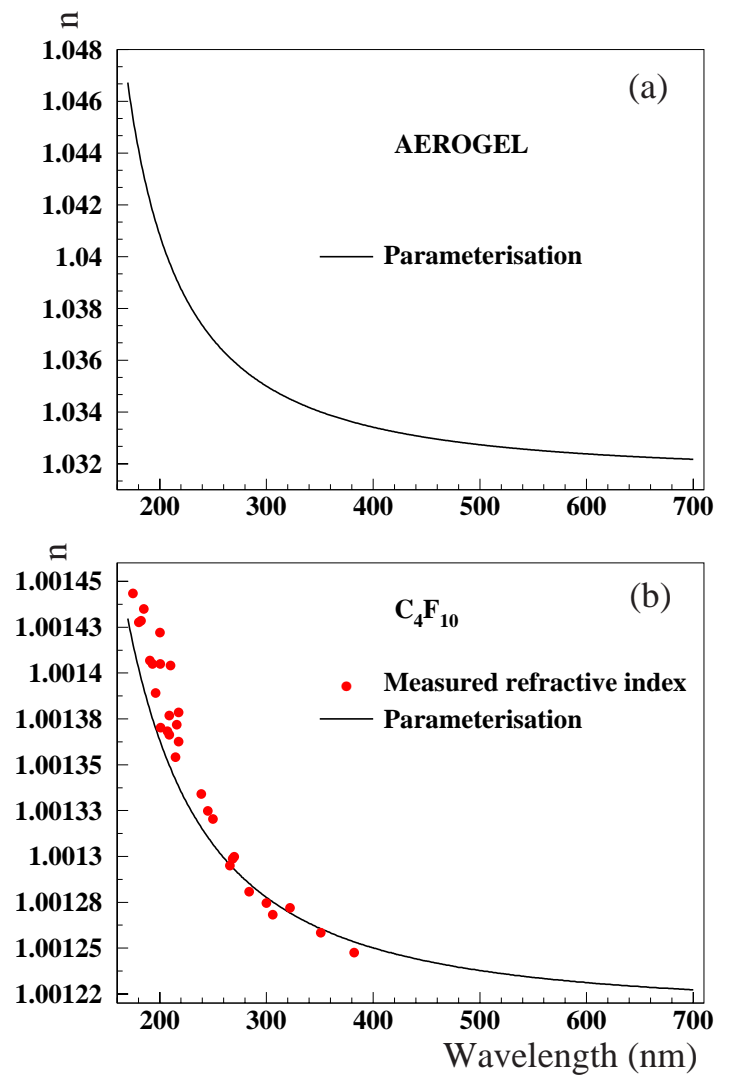

Figure 8: Parameterisations of the refractive index of aerogel and $\mathrm{C}_{4} \mathrm{~F}_{10}$ versus photon wavelength, as used by the simulation program described in the text. Datapoints shown are from [9].

a photoelectron is produced. The simulation incorporates an $18 \%$ probability of a backscattered electron being released from the silicon [10]. If a back-scattered electron is released, its flight path is calculated, assuming a uniform accelarating field of the HPD, and its new hit position recorded. Random electronic noise is also added to agree with observations in data.

- Pressure Variation of the Gas Radiator : The $\mathrm{C}_{4} \mathrm{~F}_{10}$ gas recirculation system controls the pressure to approximately \pm 20 mbar which leads to a periodic variation in the measured Cherenkov angle of approximately $0.7 \mathrm{mrad}$. This variation can be reduced substantially by deactivating the circulation system. This effectively decreases the rate of change of pressure, and allows measurements to be made over longer periods of time with greater stability.

An event display program is used to visualise the results of simulation and data events. An example of simulated events in RICH Configuration 2 is shown in Figure 9.

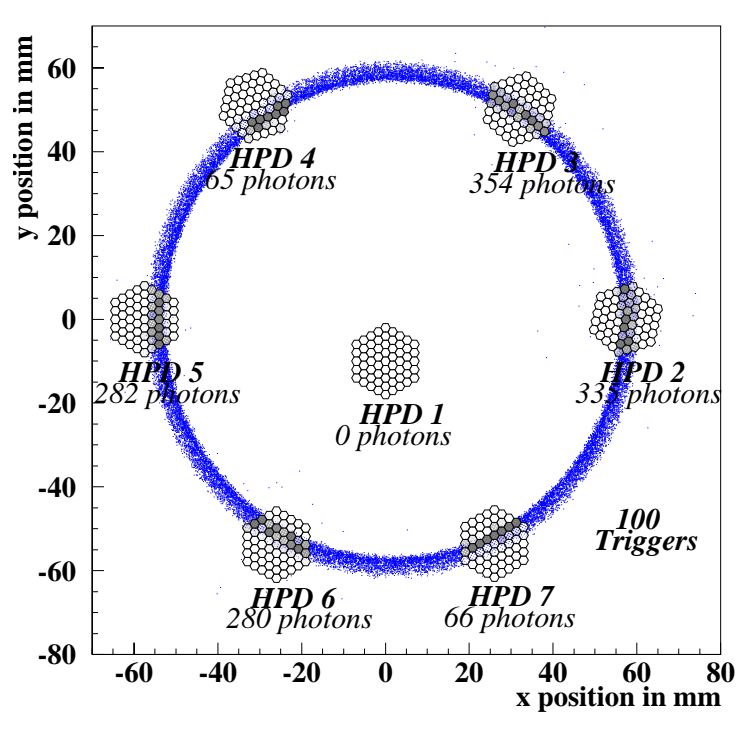

Figure 9: Event display showing the superposition of simulated events as in Configuration 2. The $x$ and $y$ axes are in units of $\mathrm{mm}$.

Contributions to the Cherenkov angle resolution for $\mathrm{RICH}$ Configuration 2 with a $\mathrm{C}_{4} \mathrm{~F}_{10}$ gas radiator are shown in Figure 10. Assuming an emission point for the photons to be the mid-point of the radiator induces a systematic contribution to the Cherenkov angle resolution shown in Figure 10(a). The effect of chromatic aberration is to induce a spread in the reconstructed values of $\theta_{c}$, and this contribution is shown in Figure 10(b). If in the reconstruction it is assumed that the beam particle follows the nominal trajectory, this results in an additional contribution to the Cherenkov angle resolution shown in Figure 10(c). Finally, the finite pixel size $\left(2 \times 2 \mathrm{~mm}^{2}\right)$ affects the reconstructed Cherenkov angle, as indicated in Figure 10(d). Here the precise nature of the modulation of the $\theta_{c}$ distribution depends critically on the relative orientations of the Cherenkov ring relative to the line of the pixels in the HPD hexagonal structure. The effects of this contribution are simulated, however the precise alignment of the photon detectors relative to the ring remains a potential source of discrepancy between data and simulation. Results are averaged over compatible HPDs, in different orientations, in order to minimise this uncertainty.

The magnification of the incident position of photons arriving at the photocathode is measured to be 1.06 from laboratory studies using a scanning LED, and is included in the simulation. Finally, background signals from electronic noise are included in the simulation by smearing the expected photoelectron signal with a Gaussian whose sigma is determined by the pedestal width observed in data. 

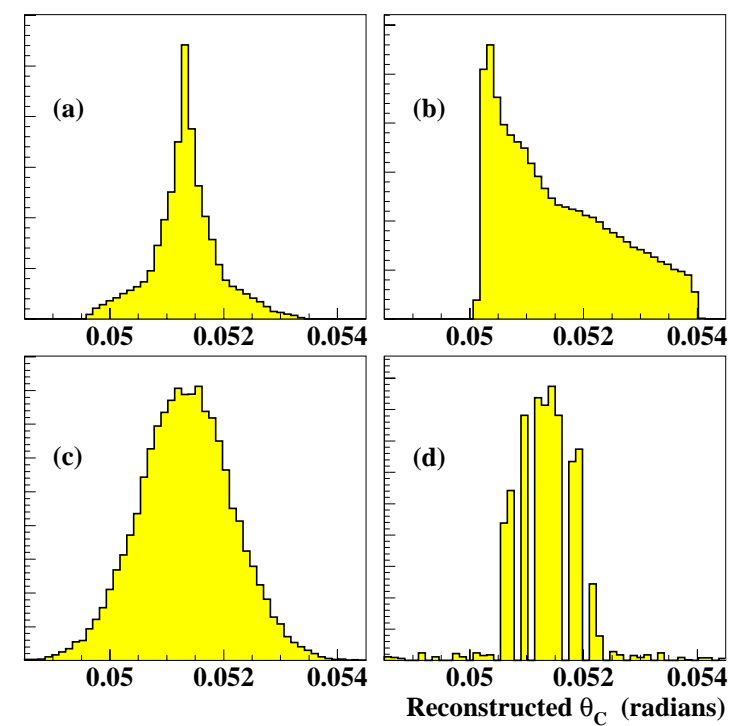

Figure 10: Plots showing examples of reconstructed $\mathrm{C}_{4} \mathrm{~F}_{10}$ Cherenkov angles when different contributions to the error are isolated in the simulation: (a) Emission point uncertainty only; (b) Chromatic aberration of the photons; (c) the particle position and direction uncertainty and; (d) the error due to the finite pixel size of $2 \times 2 \mathrm{~mm}^{2}$.

\section{$6 \quad$ Estimates of the photon yield}

The number of detected photoelectrons per event is determined for various gas and aerogel radiators, using different filters. Two aerogel samples are studied, together with air and $\mathrm{C}_{4} \mathrm{~F}_{10}$ gas radiators. Three filter configurations are tested. Either no filter is applied, or a glass or mylar filter is placed in front of the quartz input window of the HPDs.

Two complementary analyses are employed, which allow estimation of systematic uncertainties due to different methods of background subtraction, photon counting and efficiency corrections. The relevant features of the two analyses are briefly summarised here :

- Analysis 1 - Hit pixels are defined to be those with an ADC count which is greater than three pedestal sigma above the mean pedestal value for that channel. A correction is made to the observed raw photon rate when comparing with simulation, to take into account the number of signal photons which lie below this cut. This correction depends on the signal-to-noise of each channel and is estimated to represent a $15 \pm 5 \%$ loss of signal when averaged over all channels. The number of photons detected on an active pixel is estimated using a series of intervals for each pixel, which are determined using the positions and widths of the multiple peak positions observed in LED calibration spectra. Photon counts are only accepted if they lie inside a signal region, defined to be within a three pixel border around the arc observed on a given HPD. This allows the remainder of the detector region to be used to estimate background rates. These are estimated assuming that the background distribution is uniform over the whole HPD area. For gas radiators, the background correction is small, typically $\sim 5 \%$, whereas for aerogel samples it is large, between 25 and $40 \%$ depending on the filter configuration used, since the photon density is lower in this case.

- Analysis 2 - Multiple Gaussian fits are made to the observed pulse-height spectra on each pixel channel for ADC counts above a four sigma pedestal cut. By integrating the area under the signal Gaussians, and normalising to the number of recorded triggers, the numbers of photoelectrons per pixel per event are determined according to Poisson statistics. From these the total number of photoelectrons seen in all HPD's per event is obtained. Hence this analysis has no explicit background subtraction, but rather includes Rayleigh scattered photons and counts from backscattering in the total. As a consequence of the more stringent pedestal cut, contributions from electronic noise are assumed to be negligible.

Both analyses rely on simulation to correctly model the efficiency for detecting the expected number of signal photons. Analysis 1 does this explicitly, correcting the observed rate in data using factors determined from simulation. The second method includes such corrections implicitly when comparing results with simulation and assumes that the efficiencies in data and simulation are the same. Two sets of corrections are necessary. Firstly, a correction for the optical properties of the testbeam prototype, takes into account the measured values of refractive indices, aerogel clarity, transmission and reflection curves as described in Section 5. Secondly, a geometric correction is applied which takes into account losses coming from the partial detector coverage of the aergoel, air and $\mathrm{C}_{4} \mathrm{~F}_{10}$ rings. In configuration 1 these geometric efficiencies are $15 \%$ and $25 \%$ for 5 and 7 HPDs respectively for aerogel. This correction is $25 \%$ for the $\mathrm{C}_{4} \mathrm{~F}_{10}$ ring with 7 HPDs in both configurations.

\subsection{Results from photon yield analyses}

As a result of the differing Cherenkov ring radii from different radiators and experimental configurations, the number of HPDs which are used to determine the photon yield for each type varies. The layouts are specified in Table 2. The results from Analysis 1 for each of the above layouts are shown in Table 3 where they are also compared with results from simulation under the same conditions. 


\begin{tabular}{|c|c|c|c|c|}
\hline Layout & Radiator & Filter & Config. & $\begin{array}{c}\text { No. of } \\
\text { HPD } s\end{array}$ \\
\hline 1 & Air & None & $\mathbf{1}$ & 1 \\
2 & $\mathrm{C}_{4} \mathrm{~F}_{10}$ & None & $\mathbf{1}$ & 1 \\
3 & $\mathrm{C}_{4} \mathrm{~F}_{10}$ & None & $\mathbf{2}$ & 4 \\
4 & $\mathrm{C}_{4} \mathrm{~F}_{10}$ & Mylar & $\mathbf{2}$ & 2 \\
5 & Aerogel 1 & None & $\mathbf{1}$ & 4 \\
6 & Aerogel 1 & Glass & $\mathbf{1}$ & 4 \\
7 & Aerogel 1 & Mylar & $\mathbf{1}$ & 6 \\
8 & Aerogel 2 & Glass & $\mathbf{1}$ & 4 \\
\hline
\end{tabular}

Table 2: Summary of the different detector, optical and radiator configurations used to study the photon yield.

Similarly, results from Analysis 2 are summarised in

\begin{tabular}{|c|c|c|c||c|}
\hline Layout & Raw & $\begin{array}{c}\text { Backgnd. } \\
\text { Corr. }\end{array}$ & $\begin{array}{c}\text { Efficncy. } \\
\text { Corr. }\end{array}$ & $\frac{\text { Observed }}{\text { Predicted }}$ \\
\hline 1 & 4.92 & 4.56 & 4.80 & 0.90 \\
2 & 7.85 & 7.49 & 34.80 & 1.02 \\
3 & 12.83 & 12.77 & 89.46 & 1.05 \\
4 & 1.78 & 1.76 & 24.66 & 1.46 \\
\hline 5 & 1.79 & 1.31 & 11.39 & 0.73 \\
6 & 1.14 & 0.75 & 6.52 & 0.92 \\
7 & 1.34 & 0.81 & 3.71 & 0.64 \\
8 & 0.85 & 0.52 & 4.52 & 1.10 \\
\hline
\end{tabular}

Table 3: The observed, background \& efficiency corrected, and expected number of detected photoelectrons for the various radiator and filter configurations determined using Analysis 1.

Table 4. Due to differences in the numbers of pixels

\begin{tabular}{|c|c|c||c|}
\hline Layout & Observed & $\frac{\text { Observed }}{\text { Predicted }}$ & $\begin{array}{c}\text { Mean } \frac{\text { Observed }}{\text { Predicted }} \\
\text { (Anal. 1 \& 2) }\end{array}$ \\
\hline 1 & 5.06 & 0.88 & $\mathbf{0 . 8 9}$ \\
2 & 7.15 & 1.01 & $\mathbf{1 . 0 2}$ \\
3 & 12.42 & 1.10 & $\mathbf{1 . 0 8}$ \\
4 & 1.85 & 1.39 & $\mathbf{1 . 4 2}$ \\
\hline 5 & 1.80 & 0.67 & $\mathbf{0 . 7 0}$ \\
6 & 1.18 & 0.95 & $\mathbf{0 . 9 4}$ \\
7 & 1.45 & 0.90 & $\mathbf{0 . 7 7}$ \\
8 & 0.83 & 1.15 & $\mathbf{1 . 1 2}$ \\
\hline
\end{tabular}

Table 4: Observed and expected numbers of detected photoelectrons for the various radiator and filter configurations determined using Analysis 2. The final column indicates the mean of the two analyses for the ratio of Observed/Predicted numbers of photoelectrons.

used and in the pedestal selection between the two analyses, it is not possible to compare directly the raw numbers of observed photons for each layout. Instead, the combined ratio of number of photons observed to that predicted is averaged for the two methods and are given in the final column of Table 4 .

\subsection{Systematic uncertainties}

Results from the two analyses show general agreement. Larger systematic errors are expected for aerogel radiators due to the lower number of photons detected per event and the relatively large background. The differences in the ratio of observed to predicted photon yields which exist between the analyses, are used to assign a systematic uncertainty. The systematic errors are determined in terms of their effect on the mean ratio of observed to predicted photon yields and are ascribed as follows :

- Photon counting - There are clearly substantial correlations between the two analyses, hence the full size of the differences is taken as the error on the mean ratio. These vary from run to run, but on average are $4 \%$ for the gas data and $10 \%$ for the aerogel data.

- Pedestal cut - A contribution of $5 \%$ is added to cover the uncertainty in the effect of the pedestal cut. This error is conservatively assigned to all samples, although in principle it is less for the gas rings, where the fraction of hit pixels with two or more photoelectrons is significant.

- Refractive indices - There is an uncertainty in the prediction due to imprecise knowledge of the refractive indices. It is important to note that variations in the number of produced photons with changes in refractive index, are anticorrelated with the change in geometric acceptance. Therefore the resulting error is relatively small. Changes of $\pm 10 \%$ in $(n-1)$ are considered for all samples, and the effect of alternative parameterisations of the chromatic dependence are studied. All variations are found to lie within $2.5 \%$ of the number of observed photons.

- Acceptance and optical properties - Additional errors come from knowledge of the absolute geometric acceptance and from the modelling of the transmissions, reflectivity, aerogel clarity and photocathode quantum efficiency. These are estimated to result in a $7 \%$ uncertainty.

The total systematic error from the sum of all sources is $13 \%$ for the aerogel and $10 \%$ for the gas radiators.

\subsection{Discussion and detector response parameters}

This variation in the ratio of observed to predicted photon yields throughout the runs considered, is consistent with the systematic uncertainties ascribed above. Significant disagreement between 
observed and predicted photon yields occurs only for the $\mathrm{C}_{4} \mathrm{~F}_{10}$ data with the mylar-shielded HPDs. The other $\mathrm{C}_{4} \mathrm{~F}_{10}$ datasets which cover that part of the spectrum relevant for LHCb show no discrepancy. The aerogel samples give reasonable agreement thoughout, with the aerogel sample 1 being systematically, slightly lower than expectations.

It is possible to convert the average photon yields of the two analyses from Tables 3 and 4 into values of the detector response parameter [4] assuming a perfect geometrical coverage and detection efficiency. This is referred to as $N_{0}^{\text {perf }}$ and is defined here by :

$$
N_{0}^{\text {perf }}=\frac{N_{\mathrm{pe}}}{L \sin ^{2} \theta_{\mathrm{C}}}
$$

where $N_{\text {pe }}$ is the number of detected photoelectrons, corrected for backgrounds, and the effects of geometrical and threshold cut losses. A slightly modified quantity is that, taking into account the efficiency of the 3 sigma pedestal cut which is denoted by $N_{0}^{3 \sigma}$. The values of these two estimates of the detector response parameter are given in Table 5 for each of the experimental layouts used.

\begin{tabular}{|c|c|c|c|}
\hline Layout & $\theta_{\text {c }}$ (in mrad) & $N_{0}^{\text {perf }}$ & $N_{0}^{3 \sigma}$ \\
\hline 1 & 19.3 & 318.8 & 271.0 \\
2 & 50.4 & 342.8 & 291.4 \\
3 & 52.3 & 336.7 & 286.2 \\
4 & 51.2 & 91.6 & 77.8 \\
\hline 5 & 260.0 & 91.8 & 78.0 \\
6 & 258.0 & 56.8 & 48.3 \\
7 & 257.0 & 38.4 & 32.6 \\
8 & 277.0 & 55.9 & 47.5 \\
\hline
\end{tabular}

Table 5: The observed mean $\theta_{\mathrm{c}}$ and detector response parameters, with and without corrections applied for the efficiency of the 3 sigma cut on the HPD pedestal distribution.

\section{$7 \quad$ Cherenkov angle reconstruction}

Individual HPD hits are used to determine Cherenkov angles. The aim is to reconstruct the precision with which $\theta_{\mathrm{c}}$ is determined for single photons. The observed resolution in data is then compared with that expected from the simulation described in Section 5. Data are used where four HPDs, $(2,3,5,6)$, have no filter in front of their input windows, but where two HPDs, 4 \& 7, have mylar filters. The method used to reconstruct Cherenkov angles in data is illustrated in Figure 11 and requires the following parameters to be determined :

1. The detection point, $\mathbf{D}$ supplied by the physical hit position on the surface of the HPD, subject to alignment uncertainties.

2. The centre of curvature of the mirror, $\mathbf{C}$, defined by the measured focal length and the

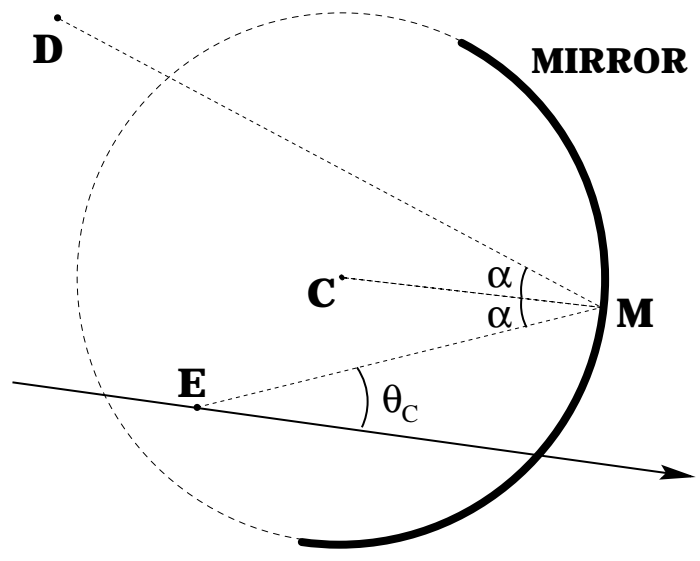

Figure 11: Schematic of the Cherenkov angle reconstruction in the tilted spherical mirror geometry.

rotation angle relative to the nominal beam direction. The focal length has a precision of $\pm 10 \mathrm{~mm}$, whereas the rotation angle is nominally 18 degrees, but is subject to small uncertainties when centering the ring image on the detector plane using micrometer screws.

3. The direction of the particle through the system determined using, either the nominal direction, $(0,0,-1)$, or an improved estimator, such as from the silicon telescope or event-byevent reconstruction.

4. The emission point, $\mathbf{E}$, of the photon is assumed to be the centre of the radiator, traversed by the particle. This is the position which minimises systematic uncertainties.

The reflection point, $\mathbf{M}$, is constrained to lie on the same plane as $\mathbf{E}, \mathbf{D}$ and $\mathbf{C}$. The two-dimensional problem is solved [11] for $\theta$ under the condition that incident and reflected angles $(\alpha)$ are equal.

\subsection{Cherenkov angle resolutions for $\mathrm{C}_{4} \mathrm{~F}_{10}$ and silica aerogel}

The small number of Cherenkov photons from the aerogel, and the absence of the silicon telescope in Configuration 1, prevents use of the improved resolution fitting methods detailed in Section 7.2. In the absence of any beam trajectory information, beyond that implied by the scintillator acceptance, this method is applied directly to data, using the nominal $(0,0,-1)$ beam direction. It serves as a cross-check to allow comparisons with more precise determinations explained later. These "raw" $\theta_{c}$ distributions are shown in Figure 12 for the case of Configuration 2 with $1 \mathrm{~m}$ of $\mathrm{C}_{4} \mathrm{~F}_{10}$. The pixellated nature of the HPD is apparent, as it induces a multi-peak structure of the $\theta_{c}$ distribution. In addition, there is a shift in the mean $\theta_{c}$, and a clear reduction in the raw resolutions for orientations of HPDs 2 and 5 where the Cherenkov ring lies parallel to a single row of pixels. 

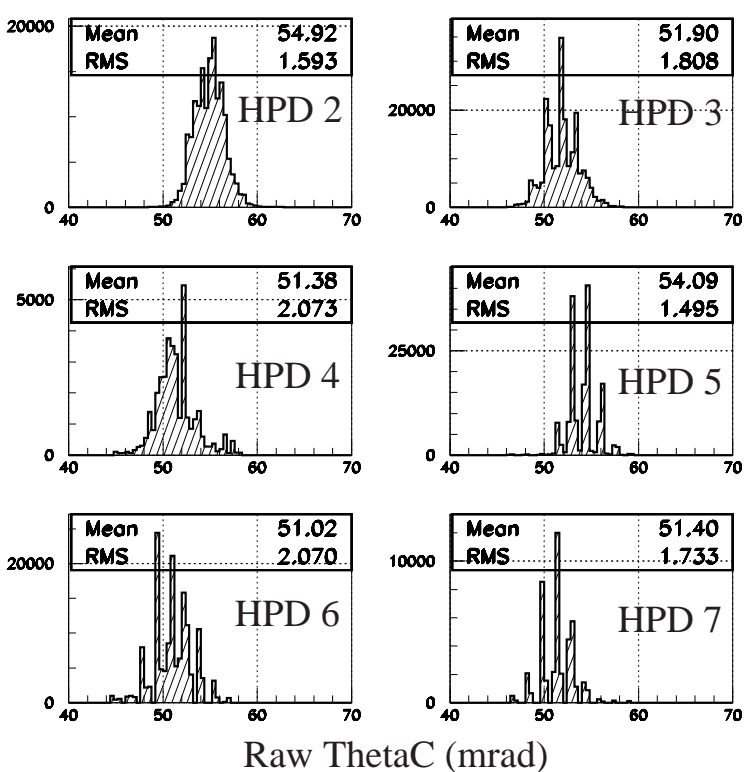

Figure 12: Raw Cherenkov angle distributions for hits detected in each of the outer HPD detectors. The $x$ axes units are in milliradians.

Raw Cherenkov resolutions are determined from data using HPDs $(2,3,5,6)$, without filters, in Figure 12, and which average $1.76 \mathrm{mrad}$. The RMS of the observed $\theta_{c}$ distribution is used as an estimate of the width, as pixellisation effects makes the distributions difficult to interpret using Gaussian fits. Similarly, the raw $\theta_{c}$ distributions are determined for runs taken with aerogel in Configuration 1 . The measured RMS values of the raw Cherenkov resolutions average $4.78 \mathrm{mrad}$ for HPDs $(2,3,5,6)$. No aerogel data was collected using Configuration 2 .

Simulation is used to determine resolution contributions due to chromatic aberration, discrete pixel sizes and the emission point uncertainty. The input beam trajectory uncertainty for this configuration is that implied by the trigger scintillator acceptance. The expected contributions are summarised in Table 6 and, when added in quadrature, total $1.72 \mathrm{mrad}$ for $\mathrm{C}_{4} \mathrm{~F}_{10}$ and $3.20 \mathrm{mrad}$ for silica aerogel. The aerogel Cherenkov angle resolution is dominated by the finite pixel size, because of the shorter focal length mirror used in Configuration 1. The agreement between observed and expected raw resolutions is reasonable considering systematic uncertainties due to estimating the widths of the discretised distributions.

In order to improve the Cherenkov angle resolution, it is necessary to reduce the dominant contributions listed in Table 6 . Effects of radiator pressure variation are reduced by deactivating the recirculation system, and measuring the mean Cherenkov angles in blocks of events recorded sequentially in time. Extrapolating the observed time variation of

\begin{tabular}{|l|r|r|}
\hline & \multicolumn{2}{|c|}{$\sigma\left(\theta_{\mathrm{c}}\right)$ (mrad) } \\
\hline Contribution Source & $\mathrm{C}_{4} \mathrm{~F}_{10}$ & Aerogel \\
\hline Pressure Variation & 0.70 & 0.70 \\
Emission Point Error & 0.58 & 0.66 \\
Chromatic Aberration & 1.03 & 1.06 \\
Finite Pixel Size & 0.56 & 2.73 \\
Beam Trajectory Error & 0.87 & 0.87 \\
\hline Total in Quadrature & 1.72 & 3.20 \\
\hline
\end{tabular}

Table 6: Summary of expected resolution contributions for the analysis of the Cherenkov angles using the nominal beam direction for $\mathrm{C}_{4} \mathrm{~F}_{10}$ without aerogel, with a mylar window, and and silica aerogel in Configurations 2 and 1 respectively.

the reconstructed Cherenkov angles allows remaining uncertainties to be extracted from data. The chromatic aberration contribution is reduced for HPDs $(4,7)$ by using mylar filters to prevent photons with wavelengths below $\sim 350 \mathrm{~nm}$ from entering the HPD photo-cathode. The beam trajectory is estimated more accurately using two methods:

- Silicon telescope data, when available, allows precise constraints on the input particle trajectory. A single specific pixel, on each of the three silicon planes, is demanded as being hit for the beam vector to be used ${ }^{5)}$.

- Geometrical reconstruction uses an elliptical fit to events with $\geq 4$ hit pixels from each of HPDs $(2,3,5,6)$ and $(4,7)$. The centre of each ellipse is used to estimate the beam trajectory for the Cherenkov angle reconstruction on that HPD. A vector, connecting the ellipse centre to the mirror centre, is reflected in the mirror to give an estimate of the incoming beam direction. Systematic checks are necessary to ensure the fit procedure does not bias the results. Resolution contribution from uncertainties in the beam trajectory can be determined from the observed error distributions from the fit. They correspond to values of $0.28 \mathrm{mrad}$ for HPDs $(2,3,5,6)$ and $0.58 \mathrm{mrad}$ for $\operatorname{HPDs}(4,7)$.

The results from data and simulated expectations are summarised separately for the two types of improved beam direction estimates.

\subsection{Improved Cherenkov angle resolutions}

Simulation is used to determine the resolution contributions for both methods, using silicon telescope (SITEL) information or the method of geometrical reconstruction (ELLIPSE). The estimated contributions from the above sources are given in Table 7. The results observed in data are summarised in Table 8 for both methods. The observed

\footnotetext{
5) To improve statistics, this constraint is relaxed slightly on the central telescope plane, by requiring that the beam pass through one of three neighbouring pixels.
} 


\begin{tabular}{|l|c|c|}
\hline & No Mylar & Mylar \\
\hline Contribution & $\sigma\left(\theta_{\mathrm{c}}\right)(\mathrm{mrad})$ & $\sigma\left(\theta_{\mathrm{c}}\right)(\mathrm{mrad})$ \\
\hline Pressure Variation & 0.02 & 0.02 \\
Emission Point & 0.58 & 0.58 \\
Chromatic Aberr. & 1.03 & 0.20 \\
Finite Pixel Size & 0.56 & 0.56 \\
\hline Beam Trajectory : & & \\
Silicon telescope & 0.38 & 0.38 \\
Elliptical Fit & 0.28 & 0.58 \\
\hline Totals : & & \\
Silicon telescope & $\mathbf{1 . 3 6}$ & $\mathbf{0 . 9 1}$ \\
Elliptical fit & $\mathbf{1 . 3 4}$ & $\mathbf{1 . 0 1}$ \\
\hline
\end{tabular}

Table 7: Summary of expected resolution contributions for the analysis of the Cherenkov angles in $\mathrm{C}_{4} \mathrm{~F}_{10}$ using silicon telescope and elliptical fit information.

\begin{tabular}{|l|c|c|c|c|}
\hline & \multicolumn{2}{|c|}{ SITEL $($ mrad $)$} & \multicolumn{2}{c|}{ ELLIPSE $($ mrad $)$} \\
\hline HPD & $\sigma\left(\theta_{\mathrm{c}}\right)$ & Mean & $\sigma\left(\theta_{\mathrm{c}}\right)$ & Mean \\
\hline 2 (no mylar) & 1.36 & & 1.25 & \\
3 (no mylar) & 1.53 & & 1.51 & \\
5 (no mylar) & 1.06 & & 1.16 & \\
6 (no mylar) & 1.42 & $\mathbf{1 . 3 4}$ & 1.68 & $\mathbf{1 . 4 0}$ \\
\hline 4 (mylar) & 0.91 & & 1.08 & \\
7 (mylar) & 1.20 & $\mathbf{1 . 0 6}$ & 1.12 & $\mathbf{1 . 1 0}$ \\
\hline
\end{tabular}

Table 8: Gaussian widths of the measured Cherenkov resolutions in data using improved beam direction estimates. This is based upon either silicon telescope information (SITEL) or geometrical reconstruction (ELLIPSE) to estimate the input beam direction for HPDs $(2,3,5,6)$ and $(4,7)$.

results using the silicon telescope (SITEL) method are generally consistent with simulations, based upon three perfectly aligned silicon detector planes with pixel dimensions of $1.3 \times 1.3 \mathrm{~mm}$. The average of the measured resolutions using this method for HPDs $(2,3,5,6)$ and $(4,7)$ are $1.34 \mathrm{mrad}$ and $1.06 \mathrm{mrad}$ respectively. The corresponding expectations from simulation are $1.36 \mathrm{mrad}$ and $0.91 \mathrm{mrad}$ respectively.

The observed Cherenkov angle distributions from geometrical reconstruction (ELLIPSE) of single photon hits are shown in Figure 13, together with Gaussian fits to the core of the distributions. The corresponding results for HPDs $(2,3,5,6)$ and $(4,7)$ are $1.40 \mathrm{mrad}$ and $1.10 \mathrm{mrad}$ respectively. These are also in good agreement with simulation, which predicts corresponding values of $1.34 \mathrm{mrad}$ and $1.01 \mathrm{mrad}$.

\subsection{Systematic cross-checks}

Results from the two analyses indicate good agreement for both HPDs with and without filters. A summary of systematic cross-checks and the

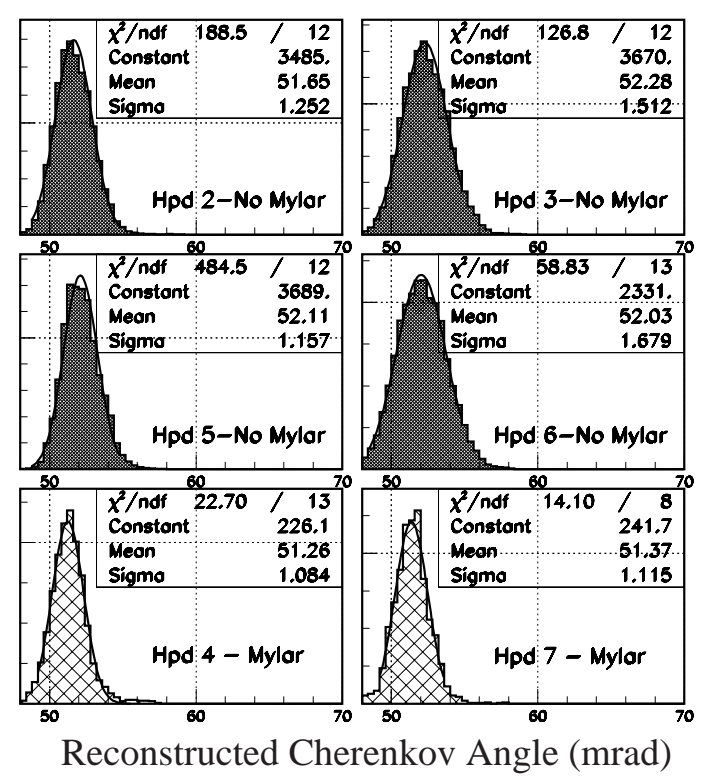

Figure 13: Measured Cherenkov angle distributions using geometrical reconstruction based on the elliptical fitting method. Results for each of the 6 HPDs are shown. The first four, $(2,3,5,6)$, are without mylar input filters and the second, (4,7), are with filters. The $x$ axes are in units of milliradians.

methods used to ascribe systematic uncertainties follows :

- HPD geometry and alignment - By studying the dependence of the mean Cherenkov angle resolution in an event as a function of the number of photons, an estimate of the residual misalignment of the detector prototype is determined. This corresponds to between 0.1 and $0.3 \mathrm{mrad}$ for HPDs $(2,3,5,6)$ and $(4,7)$ respectively.

- Radiator pressure variation - The resolutions given above are determined from a subset of the data within which the pressure variation of the mean Cherenkov angle is determined to be $0.02 \mathrm{mrad}$.

- Beam composition - The beam contamination cut on the upstream Cherenkov counter is varied widely by changing the cut applied on the output from the upstream Cherenkov counter. The maximum effect observed on the mean Cherenkov angle resolution is $0.1 \mathrm{mrad}$.

- Noise contamination from the hit selection - The effect of the default 4 sigma selection cut is studied by varying it from 3 to 5 sigma and determining the shift in the average Cherenkov resolution. The shift is asymmetric due to the increasing noise contamination when the cut is reduced to 3 sigma. As a conservative value, the systematic error is estimated to be $0.1 \mathrm{mrad}$. 
- Use of the geometrical fitting procedure - To check for a systematic bias in the geometrical fitting method, a similar selection of HPDs $(4,7)$ is applied to that for $(2,3,5,6)$ in order to verify the stability of the measured resolutions. The procedure followed is to use hits from HPDs 3 \& 6 only. This is the same but diametrically opposite configuration to HPDs $4 \& 7$. The hit multiplicities of $(3,6)$ are then reduced to those of $(4,7)$ by randomly deleting hits, to emulate the effects of the mylar coverings on HPDs $(4,7)$. The results indicate that the effects of the elliptical fitting method induce a maximum change in the observed Cherenkov angle resolution of 0.08 mrad.

When combined with the total uncertainties, listed above, the systematic error on the measured Cherenkov resolution is expected to be of the order of between \pm 0.2 and \pm 0.3 mrad for HPDs $(2,3,5,6)$ and $(4,7)$ respectively.

\section{Summary and conclusions}

A prototype of the $\mathrm{RICH}-1$ detector has been constructed, and operated in the CERN T9 test beam. Focussed ring images from pions, kaons and protons are detected on a plane of HPD's, using silica aerogel, air and $\mathrm{C}_{4} \mathrm{~F}_{10}$ gaseous radiators. The number of detected Cherenkov photons and their measured spatial precision were studied.

The number of detected photons are measured both in data and in simulated event samples. The number of photons observed for both gas and aerogel radiators is found to be in good agreement between data and simulation. The resolution with which the Cherenkov angles are reconstructed is determined and compared with simulation. The expected resolution contributions are in good agreement with their observed values. Both the photon yields and Cherenkov angle resolutions observed in data satisfy the requirements of the proposed $\mathrm{RICH}$ 1 detector, as part of the LHCb experiment.

Future work in intended to study the properties of the LHCb RICH-2 detector [12]. This uses an increased focal-length mirror and a single $\mathrm{CF}_{4}$ gas radiator, to obtain an expected angular resolution of $0.35 \mathrm{mrad}$ [1]. This will allow particle indentification to be extended from the upper limit of $65 \mathrm{GeV} / \mathrm{c}$ of RICH-1 to approximately $100 \mathrm{GeV} / \mathrm{c}$. It is also planned to use the $\mathrm{RICH}$ prototype system to test various types of new photodetectors currently under development [13].

\section{$9 \quad$ Acknowledgements}

The work reported here would not have been possible without the technical support provided by colleagues from our home institutes. We thank, in particular, D.Campbell, C.Raine, I.Clark, P.Evans, S.Greenwood, H.Baughan, S.Johnson, V.Kasey,
R.Knott, and M.Rankin for their contributions to the construction of the prototype RICH and its associated gas system and read-out electronics. Colleagues from the LHCb Collaboration have provided valuable advice and assistance during the beam tests at CERN, especially, R.Forty, G.McEwen, O.Ullaland and T.Ypsilantis. The silicon beam telescope was provided for us as a working system by E.Chesi and J.Seguinot. E.Nappi provided some of the aerogel samples and advised on its handling. Finally, we gratefully acknowledge the CERN PS division for their provision of the test beam and the UK Particle Physics and Astronomy Research Council for their financial support.

\section{References}

[1] LHCb Technical Proposal, CERN/LHCC 98-4, Feb. 1998.

[2] R.Forty, Nucl. Instr. and Meth. A 384 (1996) 167.

[3] E. Albrecht et al., Nucl. Instr. and Meth. A 411 (1998) 249.

[4] T.Ypsilantis and J.Seguinot, Nucl Instr. and Meth. A 368 (1995) 229.

[5] O. Toker et al., Nucl. Instr. and Meth. A 340 (1994) 572.

[6] T.Ypsilantis and J.Seguinot, Nucl Instr. and Meth. A 343 (1994) 30.

[7] P.W. Langhoff and M. Karplus, The Optical Society of America, Volume 59, Number 7, p. 863, July 1969.

[8] R. Abjean et al, Nucl. Instr. and Meth. A, 354 (1995) 417.

[9] Private Communication with E. Fokitis from CERN EP/LE Division. Also see http://home.cern.ch/d/delrich/www /notes/rich98ntua.ps.

[10] E. Darlington, J. Phys. D8 (1975) 85.

[11] R. Forty, Nucl. Instr. and Meth. A 384 (1996) 167.; the term $4 a\left(b^{2}+C^{2}\right) R$ in equation (1) should read $4 a\left(b^{2}+C^{2}\right)$.

[12] E. Albrecht et al., Submitted as an accompanying article to Nucl Instr. and Meth. A in November 1999.

[13] E. Albrecht et al., Nucl. Instr. and Meth. A 433 (1999) 15. 